\title{
Principales causas de muerte de agricultores en Colombia periodo 2010-2016
}

\section{Leading causes of deaths among farmers in Colombia 2010 -2016}

\author{
Ivan-Andrés Trujillo-Abella'; Cristian-Felipe Orjuela-Yacué ${ }^{1}$
}

Forma de citar: Trujillo-Abella IA; Orjuela-Yacué CF. Principales causas de muerte de agricultores en Colombia periodo 20102016, Salud UIS. 2020; 52(1): 41-49. doi. http://dx.doi.org/10.18273/revsal.v52n1-2020006 @c) (1)

\section{Resumen}

Introducción: En la literatura, los agricultores presentan mayor mortalidad por algunos tipos de cáncer y causas externas. Sin embargo, los resultados entre estudios no son concluyentes, debido a las diferencias metodológicas, como por las heterogeneidades implícitas en las actividades desarrolladas por los individuos en los diferentes sectores agrícolas y unidades geográficas. Objetivo: Describir las principales causas de muerte de los agricultores en Colombia para el periodo 2010 - 2016, e identificar si la ocupación en sí misma es un factor de riesgo para la mortalidad por dichas causas. Metodología: Se utilizaron los certificados de defunción para establecer la causa de muerte de los individuos, y se estimó por medio de regresión logística odds ratios ajustados por variables socioeconómicas como medida del riesgo ocupacional. Resultados: Los agricultores presentan mayor riesgo de mortalidad por neoplasias (OR; 1.14 IC 95\% 1.12-1.71), por enfermedades del sistema circulatorio (OR; 1.030 IC 95\% 1.01-1.04), y por signos y síntomas mal definidos (OR; 1.22 IC 95\% 1.15 - 1.30). Discusión: Los resultados pueden estar sesgados por los errores habituales de los estudios retrospectivos. Sin embargo, las limitaciones podrían suscribirse a diferencias numéricas del riesgo y no de su significancia, por lo que los hallazgos de este trabajo serían consistentes con la presencia del riesgo más que con su magnitud. Conclusiones: Para establecer con mayor precisión el riesgo ocupacional de los agricultores colombianos, se requieren esfuerzos institucionales y estudios prospectivos como los realizados por la AHS en Estados Unidos.

Palabras clave: Agricultor; Agricultura; Muerte; Riesgo; Neoplasia; Cáncer.

\begin{abstract}
Introduction: In the liturature, farmers have higher mortality from some type of cancer and external causes. However, the results are not conclusive, due to differential approaches and methodologies used in the studies, and differences in the activities developed by farmers in the geographical areas. Objective: Describe the main causes of death of farmers in Colombia from 2010 to 2016, and identify if the occupation itself is a risk factor for mortality. Methodology: We used odds ratios adjusted by logistic regression and socioeconomics variables as a measure of occupational risk in each cause of death. Death certificates were used to establish underlying cause of death. Results: Farmers have higher risk of die by neoplasm (OR; 1.14 CI 95\% 1.12-1.71), circulatory system diseases
\end{abstract}

1. Universidad Surcolombiana. Neiva, Colombia.

Correspondencia: Iván Trujillo. Dirección: Calle 9 No. 15-25 Teléfono: (+8) 8718310. Correo electrónico: ivan.trujillo@usco.edu.co 
(OR; 1.030 IC 95\% 1.01-1.04), and missdefinition disease (OR; 1.22 IC 95\% 1.15 - 1.30). Discussion: Our results could be biased, due the retrospective design. Nonetheless, these results could be consistent with the existence of risk more than magnitude itself. Conclusions: To have a major precision in the risk estimation is necessary institutional efforts to make prospective studies as AHS in United States.

Keywords: Farmer; Agriculture; Death; Risk; Neoplasm; Cancer.

\section{Introducción}

En la literatura, los agricultores presentan mayor mortalidad por algunos tipos de cáncer y causas externas. Sin embargo, los resultados no son concluyentes, debido a diferencias metodológicas, así como por las heterogeneidades implícitas en las actividades desarrolladas, por los individuos en los diferentes sectores agrícolas y unidades geográficas ${ }^{1,2}$.

Los tipos de cáncer usualmente asociados con la profesión agrícola son; linfoma No-hodking ${ }^{3}$, cáncer de cerebro ${ }^{4}$ y próstata ${ }^{5,6}$; que resultan de igual forma, estar asociados con una amplia gama de sustancias y factores de riesgo a los que están expuestos los agricultores como pesticidas, químicos fertilizantes, solventes e intensidad de rayos UV, entre otros? Las causas externas de mayor mortalidad para los agricultores son las relacionadas con accidentes laborales y el suicidio ${ }^{8-11}$. Para Colombia, no se han identificado las principales causas de muerte del grupo agricultor, y tampoco si la profesión en si misma está asociada con diferencias en el perfil de mortalidad con respecto a otros grupos ocupacionales. Por lo que, describir las principales causas de muerte de los agricultores en Colombia y determinar si la ocupación en sí, es un factor de riesgo inherente para la mortalidad por dichas causas es el principal objetivo de este trabajo.

\section{Metodología}

Para el siguiente trabajo, se utilizaron el total de certificados de defunción en Colombia para el periodo 2010-2016, disponibles en el sitio oficial del Departamento Administrativo Nacional de Estadística (DANE). En algunos países, la ocupación en los certificados de defunción se registra teniendo en cuenta el International Standard Classifications of Occupations (ISCO). Sin embargo, para el periodo de estudio, la actividad u ocupación de los individuos no se encontraba homogenizada, por lo que la identificación de los grupos ocupacionales; agricultores, inactivos y sin información se realizó por medio de un algoritmo, que combinó diferentes cadenas de caracteres para cada una de las ocupaciones. Igualmente, se clasificaron como inactivos los adultos mayores, desempleados, y/o pensionados, con el objetivo de controlar el efecto de "Healthy Worker Effect"12-14 debido a la posible inferioridad de la actividad física de los individuos en esta categoría en comparación con el grupo agricultor. Los individuos sin información se definieron como aquellos que no presentaban ocupación o que tenían como máximo dos caracteres registrados en la ocupación. Los demás individuos, fueron definidos como no agricultores. Cabe señalar que el ejercicio fue rectificado por los autores para reducir errores de clasificación.

Para las causas de muerte se definieron en siete categorías (enfermedades transmisibles, neoplasias, enfermedades del sistema circulatorio, afecciones perinatales, causas externas, demás causas y signos y síntomas mal definidos) usando la lista 6-67 de la Organización Panamericana de la Salud (OPS) que agrupa las enfermedades (en tres dígitos) con base en el código CIE-10, se identificaron las respectivas causas básicas de muerte, que en últimas son las patologías o incidentes precursores de los hechos o demás eventos que desencadenan la muerte del individuo.

Para los análisis se tuvieron en cuenta las variables como el sexo, la edad definida en rangos ( $<1$ año, 1-4 años, 5-14 años, 15-44 años, 45-64 años, mayores e iguales a 65 años), la escolaridad definida como con algún nivel educativo o ninguno, el área de residencia (cabecera municipal, centro poblado, y rural disperso) y el régimen de seguridad social (contributivo, subsidiado, excepción, especial, y no asegurado). Cabe señalar, que todas las variables anteriormente mencionadas presentan una categoría para determinar la falta de información.

Como medida de riesgo ocupacional se estimaron los Odds Ratios (ORs) para las siete (7) principales causas ajustados por variables socioeconómicas, por medio de regresión logística incondicional, teniendo como referencia todos aquellos individuos que habían fallecido por cualquiera de las demás 
causas. La estimación del riesgo se llevó a cabo para los individuos que presentaban la información de las variables socioeconómicas completa (muestra) y para los que no (submuestra) con el fin de evitar sesgos de categorización.

\section{Resultados}

Para el periodo de estudio (2010-2016) se registraron en Colombia 1,451,775 defunciones, siendo la principal causa de muerte las enfermedades del sistema circulatorio. Como se evidencia en la Tabla 1, estas representan aproximadamente el 31\% de las muertes totales. Las demás causas, con un $25 \%$ de las muertes totales son la segunda causa de muerte en la población, siguiendo las neoplasias y las causas externas con una participación de $19 \%$ y $14 \%$ respectivamente. Cabe destacar la baja participación de aproximadamente $7 \%$ de las enfermedades transmisibles en el total de las muertes para el periodo de estudio, lo que contrasta con la denominada transición epidemiológica ${ }^{15,16}$.

La principal causa de muerte poblacional, es también la principal causa de muerte entre los grupos ocupacionales; agricultores, no agricultores e inactivos. Siendo mayor el porcentaje de muertes por enfermedades del sistema circulatorio en el grupo agricultor que en las otras ocupaciones. No obstante, en el grupo sin información ocupacional la principal causa de muerte son las causas externas, teniendo una diferencia de $2.5 \%$ porcentuales por encima de las causas del sistema circulatorio, que es la segunda causa de muerte en dicho grupo.

En el total de la muestra, se evidenció mayor proporción de individuos del sexo masculino, con una diferencia aproximada de 12,2 puntos porcentuales con respecto al sexo opuesto. Cabe resaltar que existe una diferencia significativa en la proporción de individuos que en la muestra corresponden al sexo masculino en comparación con el sexo femenino, tanto para el grupo agricultor, como para los individuos inactivos. No obstante, el grupo no agricultor fue el único que registró mayor proporción de defunciones del sexo femenino.

De manera general, para las otras características socioeconómicas de la Tabla 1 se encuentra que el grupo etario con mayor mortalidad es de 65 años o más. El grupo ocupacional (sin contar a los que no tienen información ocupacional) más alfabetizado es el grupo inactivo, seguido de los no agricultores, y el grupo agricultor donde la menor proporción de individuos tiene algún nivel educativo. Igualmente, para el grupo agricultor en comparación con los otros grupos ocupacionales, una considerable proporción no vivía en la cabecera, y la mayor parte pertenecía al régimen subsidiado.

Para determinar si la ocupación es un factor de riesgo inherente en las causas específicas de mortalidad, se excluyeron del análisis todos aquellos individuos que no presentaban información en alguna de las variables socioeconómicas, con el fin de evitar sesgos de categorización. Posteriormente, se determinó si existía sesgo de selección como se muestra en la Tabla 2.

Lo que sugiere la Tabla 2, es que existe sesgo de selección por todas las características analizadas y que los individuos con los que contamos para el análisis de riesgo difieren sistemáticamente del grupo excluido. Sin embargo, como se mencionó anteriormente, el cálculo de los ORs se llevó a cabo teniendo en cuenta, la muestra total y la submuestra (individuos con información incompleta) para contrastar los resultados. Cabe señalar, que la principal limitación del sesgo de selección, es que los resultados no pueden ser extrapolables a la población en general, pues se considera que los individuos son sistemáticamente diferentes.

Las principales diferencias entre los individuos excluidos y no excluidos, se encuentran en la proporción de individuos cuya causa básica de muerte fueron las neoplasias, las enfermedades del sistema circulatorio y las causas externas. Los grupos de edad y la escolaridad también presentan diferencias significativas, siendo relativamente de mayor edad el grupo no excluido, como también más alfabetizado en comparación con los individuos que no contaban con la información completa.

La siguiente tabla presenta los ORs ajustados por las variables socioeconómicas anteriormente descritas (sexo, grupo de edad, escolaridad, área de residencia habitual del individuo, y régimen de seguridad social) y por el departamento habitual de residencia del individuo; tanto en la muestra como la submuestra utilizando como grupo de referencia el grupo no agricultor. 
Tabla 1. Causas de muerte y características sociodemográficas de las defunciones en Colombia por grupos ocupacionales (2010- 2016).

\begin{tabular}{|c|c|c|c|c|c|c|c|c|c|c|}
\hline & \multicolumn{10}{|c|}{ Profesión } \\
\hline & \multicolumn{2}{|c|}{ Agricultor } & \multicolumn{2}{|c|}{ Inactivo } & \multicolumn{2}{|c|}{ No- agricultor } & \multicolumn{2}{|c|}{ Sin información } & \multicolumn{2}{|r|}{ Total } \\
\hline & $\%$ & CI 95\% & $\%$ & CI 95\% & $\%$ & CI 95\% & $\%$ & CI 95\% & $\%$ & CI 95\% \\
\hline \multicolumn{11}{|l|}{ Causas de muerte } \\
\hline $\begin{array}{l}\text { Enfermedades transmisibles } \\
(\mathrm{n}=97,621)\end{array}$ & 5,4 & {$[5,2-5,5]$} & 6,2 & {$[6,1-6,4]$} & 7,2 & {$[7,2-7,3]$} & 6,3 & {$[6,3-6,4]$} & 6,7 & {$[6,7-6,8]$} \\
\hline Neoplasias $(n=276,203)$ & 19,1 & {$[18,9-19,4]$} & 23,5 & {$[23,2-23,8]$} & 23,3 & {$[23,2-23,3]$} & 12,2 & {$[12.1-12.2]$} & 19,0 & {$[19,0-19,1]$} \\
\hline Sistema Circulatorio $(\mathrm{n}=448,398)$ & 38,1 & {$[37,8-38,4]$} & 34,4 & {$[34,0-34,8]$} & 33,8 & {$[33,7-33,9]$} & 24,5 & {$[24,3-24,6]$} & 30,9 & {$[30,8-31,0]$} \\
\hline Afecciones perinatales $(n=27,698)$ & 0,0 & & 0,0 & {$[0,0-0,0]$} & 0,0 & {$[0,0-0,0]$} & 5,4 & {$[5,3-5,5]$} & 1,9 & {$[1,9-1,9]$} \\
\hline Causas externas $(n=209,299)$ & 10,9 & {$[10,7-11,1]$} & 6,3 & {$[6,1-6,5]$} & 7,1 & {$[7,1-7,2]$} & 27,1 & {$[27,0-27,2]$} & 14,4 & {$[14,4-14,5]$} \\
\hline Demás causas $(\mathrm{n}=363,318)$ & 24,4 & {$[24,1-24,6]$} & 28,6 & {$[28,3-29,0]$} & 27,3 & {$[27,2-27,4]$} & 21,4 & {$[21,3-21,5]$} & 25,0 & {$[25,0-25,1]$} \\
\hline $\begin{array}{l}\text { Signos, síntomas y afecciones mal } \\
\text { definidas }(n=29,189)\end{array}$ & 2,1 & {$[2,0-2,2]$} & 1,0 & {$[0,9-1,0]$} & 1,3 & {$[1,3-1,4]$} & 3,1 & {$[3,1-3,2]$} & 2,0 & {$[2,0-2,0]$} \\
\hline Sin información $(n=49)$ & 0,0 & & 0,0 & {$[0,0-0,0]$} & 0,0 & {$[0,0-0,0]$} & 0,0 & {$[0,0-0,0]$} & 0,0 & {$[0,0-0,0]$} \\
\hline Total $(n=1,451,775)$ & 100,0 & & 100,0 & & 100,0 & & 100,0 & & 100,0 & \\
\hline Pearson: Uncorrected chi2 $(21)=$ & $1,81 \mathrm{e}+05$ & & & & & & & & & \\
\hline Design-based $F(21.00,3.05 \mathrm{e}+07)=$ & $86,419,061$ & $\operatorname{Pr}=$ & 0,000 & & & & & & & \\
\hline \multicolumn{11}{|c|}{ Sexo } \\
\hline Masculino $(\mathrm{n}=814,798)$ & 91,7 & {$[91,5-91,8]$} & 80,6 & {$[80,3-80,9]$} & 42,8 & {$[42,7-42,9]$} & 64,6 & {$[64,5-64,7]$} & 56,1 & {$[56,0-56,2]$} \\
\hline Femenino $(n=636,763)$ & 8,3 & {$[8,2-8,5]$} & 19,4 & {$[19,1-19,7]$} & 57,2 & {$[57,1-57,3]$} & 35,4 & {$[35,2-35,5]$} & 43,9 & {$[43,8-43,9]$} \\
\hline indeterminado $(\mathrm{n}=214)$ & 0,0 & {$[0,0-0,0]$} & 0,0 & & 0,0 & {$[0,0-0,0]$} & 0,0 & {$[0,0-0,0]$} & 0,0 & {$[0,0-0,0]$} \\
\hline Total $(n=1,451,775)$ & 100,0 & & 100,0 & & 100,0 & & 100,0 & & 100,0 & \\
\hline Pearson: Uncorrected chi2(6) $=$ & $1,47 \mathrm{e}+05$ & & & & & & & & & \\
\hline Design-based $F(6.00,8.71 \mathrm{e}+06)=$ & $2,45 \mathrm{e}+04$ & $\operatorname{Pr}=$ & 0,000 & & & & & & & \\
\hline \multicolumn{11}{|l|}{ Grupos de edad } \\
\hline$<1$ año $(\mathrm{n}=54,398)$ & 0,0 & & 0,0 & & 0,0 & {$[0,0-0,0]$} & 10,7 & {$[10,6-10,7]$} & 3,7 & {$[3,7-3,8]$} \\
\hline $1-4$ años $(n=11,587)$ & 0,0 & & 0,0 & & 0,0 & {$[0,0-0,0]$} & 2,3 & {$[2,2-2,3]$} & 0,8 & {$[0,8-0,8]$} \\
\hline $5-14$ años $(n=15,076)$ & 0,0 & {$[0,0-0,1]$} & 0,1 & {$[0,1-0,1]$} & 1,0 & {$[1,0-1,0]$} & 1,4 & {$[1,4-1,4]$} & 1,0 & {$[1,0-1,1]$} \\
\hline $15-44$ años $(n=225,747)$ & 9,9 & {$[9,7-10,1]$} & 4,0 & {$[3,8-4,1]$} & 11,9 & {$[11,8-12,0]$} & 23,7 & {$[23,6-23,9]$} & 15,5 & {$[15,5-15,6]$} \\
\hline $45-64$ años $(n=281,971)$ & 18,6 & {$[18,4-18,8]$} & 12,8 & {$[12,5-13,0]$} & 21,3 & {$[21,2-21,4]$} & 17,7 & {$[17,6-17,8]$} & 19,4 & {$[19,4-19,5]$} \\
\hline 65 años o mayores $(n=860,148)$ & 71,5 & {$[71,2-71,7]$} & 83,1 & {$[82,9-83,4]$} & 65,8 & {$[65,7-65,9]$} & 43,7 & {$[43,6-43,9]$} & 59,2 & {$[59,2-59,3]$} \\
\hline Edad desconocida $(n=2,848)$ & 0,0 & {$[0,0-0,0]$} & 0,0 & {$[0,0-0,0]$} & 0,0 & {$[0,0-0,0]$} & 0,5 & {$[0,5-0,5]$} & 0,2 & {$[0,2-0,2]$} \\
\hline Total $(\mathrm{n}=1,451,775)$ & 100,0 & & 100,0 & & 100,0 & & 100,0 & & 100,0 & \\
\hline Pearson: Uncorrected chi2(18) $=$ & $2,04 \mathrm{e}+05$ & & & & & & & & & \\
\hline Design-based $F(17,00,2,47 \mathrm{e}+07)=$ & $1,20 \mathrm{e}+04$ & $\operatorname{Pr}=$ & 0,000 & & & & & & & \\
\hline
\end{tabular}

\section{Escolaridad}

Algún nivel educativo $(\mathrm{n}=854,613) \quad 56,8 \quad[56,6-57,1] \quad 80,6 \quad[80,3-80,9] \quad 67,5 \quad[67,4-67,6] \quad 43,7 \quad[43,6-43,9] \quad 58,9 \quad[58,8-58,9]$ Sin educación $(\mathrm{n}=254,556)$

Sin información $(n=342,606)$

Total $(n=1,451,775)$

$24,4 \quad[24,2-24,7] \quad 4,8 \quad[4,6-5,0] \quad 14,2[14,1-14,3] 22,4 \quad[22,3-22,5] \quad 17,5[17,5-17,6]$

\begin{tabular}{lccccc} 
Total $(\mathrm{n}=1,451,775)$ & 100,0 & 100,0 & 100,0 & 100,0 & 100,0 \\
\hline Pearson: Uncorrected chi2 $(6)=$ & $9,07 \mathrm{e}+04$ & & & & \\
Design-based $\mathrm{F}(6,00,8,71 \mathrm{e}+06)=$ & $1,51 \mathrm{e}+04$ & $\operatorname{Pr}=$ & 0,000 & &
\end{tabular}

\section{Area de residencia}

Cabecera Municipal $(\mathrm{n}=1,159,868) \quad 49,3 \quad[49,1-49,6] \quad 93,7 \quad[93,5-93,9] \quad 84,8 \quad[84,7-84,8] \quad 78,1 \quad[78,0-78,2] \quad 79,9 \quad[79,8-80,0]$

Centro Poblado $(\mathrm{n}=80,722)$

Rural disperso $(\mathrm{n}=188,922)$

Sin información $(\mathrm{n}=22,263)$

Total $(n=1,451,775)$

Pearson: Uncorrected chi2(9) =

11,4

39,1

0,2

100,0

Design-based F $(9,00,1,31 \mathrm{e}+07)$

\section{Regimen de seguridad social}

Contributivo $(\mathrm{n}=527,421)$

Subsidiado $(\mathrm{n}=708,197)$

Excepción $(\mathrm{n}=44,439)$

Especial $(n=4,998)$

No asegurado $(n=122,725)$

Sin información $(n=43,995)$

Total $(\mathrm{n}=1,451,775)$

Pearson: Uncorrected chi2(15) =

$1,39 \mathrm{e}+04$

$\begin{array}{lllllllll}{[11,2-11,5]} & 2,1 & {[2,0-2,2]} & 4,6 & {[4,6-4,7]} & 6,1 & {[6,0-6,1]} & 5,6 & {[5,5-5,6]}\end{array}$

$[38,8-39,4] \quad 3,8 \quad[3,7-4,0] \quad 10,3 \quad[10,2-10,4] \quad 12,1 \quad[12,0-12,2] \quad 13,0 \quad[13,0-13,1]$

$\begin{array}{ccccccccc}{[0,2-0,2]} & 0,3 & {[0,3-0,4]} & 0,3 & {[0,3-0,4]} & 3,8 & {[3,7-3,8]} & 1,5 & {[1,5-1,6]} \\ 100,0 & & 100,0 & & 100,0 & & 100,0 & \end{array}$

Design-based $\mathrm{F}(15,00,2,18 \mathrm{e}+07)=1,40 \mathrm{e}+04 \quad \operatorname{Pr}=0,000$

Fuente: Elaboración propia con información del DANE. 
Principales causas de muerte de agricultores en Colombia periodo 2010-2016

Tabla 2. Características de los individuos en la muestra con y sin información completa para la determinación del sesgo de selección y la estimación de los ORs.

\begin{tabular}{|c|c|c|c|c|c|c|}
\hline & \multicolumn{6}{|c|}{ Selección } \\
\hline & \multicolumn{2}{|c|}{ No excluido } & \multicolumn{2}{|c|}{ Excluido } & \multicolumn{2}{|r|}{ Total } \\
\hline & $\%$ & CI 95\% & $\%$ & CI 95\% & $\%$ & CI 95\% \\
\hline \multicolumn{7}{|l|}{ Causas de muerte } \\
\hline Enfermedades transmisibles $(\mathrm{n}=97,621)$ & 6,7 & {$[6,6-6,7]$} & 6,8 & {$[6,7-6,9]$} & 6,7 & {$[6,7-6,8]$} \\
\hline Neoplasias $(n=276,203)$ & 24,1 & {$[24,0-24,1]$} & 13,4 & {$[13,3-13,5]$} & 19,0 & {$[19,0-19,1]$} \\
\hline Sistema Circulatorio $(\mathrm{n}=448,398)$ & 34,4 & {$[34,3-34,5]$} & 27,0 & {$[26,9-27,1]$} & 30,9 & {$[30,8-31,0]$} \\
\hline Afecciones perinatales $(n=27,698)$ & 0,0 & {$[0,0-0,0]$} & 4,0 & {$[4,0-4,1]$} & 1,9 & {$[1,9-1,9]$} \\
\hline Causas externas $(n=209,299)$ & 6,9 & {$[6,9-7,0]$} & 22,8 & {$[22,7-22,9]$} & 14,4 & {$[14,4-14,5]$} \\
\hline Demas causas $(\mathrm{n}=363,318)$ & 26,7 & {$[26,6-26,8]$} & 23,1 & {$[23,0-23,2]$} & 25,0 & {$[25,0-25,1]$} \\
\hline Signos, sintomas y afecciones mal definidas $(n=29,189)$ & 1,2 & {$[1,2-1,3]$} & 2,9 & {$[2,8-2,9]$} & 2,0 & {$[2,0-2,0]$} \\
\hline Sin información $(n=49)$ & 0,0 & & 0,0 & {$[0,0-0,0]$} & 0,0 & {$[0,0-0,0]$} \\
\hline Total $(\mathrm{n}=1,451,775)$ & 100,0 & & 100,0 & & 100,0 & \\
\hline Pearson: Uncorrected chi2 $(7)=$ & $1,29 \mathrm{e}+05$ & & & & & \\
\hline Design-based $F(7,00,1,02 \mathrm{e}+07)=$ & $1,84 \mathrm{e}+04$ & $\operatorname{Pr}=$ & 0,000 & & & \\
\hline \multicolumn{7}{|l|}{ Sexo } \\
\hline Masculino $(\mathrm{n}=814,798)$ & 51,4 & {$[51,3-51,5]$} & 61,5 & {$[61,3-61,6]$} & 56,1 & {$[56,0-56,2]$} \\
\hline Femenino $(n=636,763)$ & 48,6 & {$[48,5-48,7]$} & 38,5 & {$[38,4-38,6]$} & 43,9 & {$[43,8-43,9]$} \\
\hline indeterminado $(\mathrm{n}=214)$ & 0,0 & & 0,0 & {$[0,0-0,0]$} & 0,0 & {$[0,0-0,0]$} \\
\hline Total $(n=1,451,775)$ & 100,0 & & 100,0 & & 100,0 & \\
\hline Pearson: Uncorrected chi2 $(2)=$ & $1,52 \mathrm{e}+04$ & & & & & \\
\hline Design-based $\mathrm{F}(2,00,2,90 \mathrm{e}+06)=$ & $76,235,900$ & $\operatorname{Pr}=$ & 0,000 & & & \\
\hline \multicolumn{7}{|l|}{ Grupos de edad } \\
\hline$<1$ año $(\mathrm{n}=54,398)$ & 0,0 & {$[0,0-0,0]$} & 8,0 & {$[7,9-8,0]$} & 3,7 & {$[3,7-3,8]$} \\
\hline $1-4$ años $(\mathrm{n}=11,587)$ & 0,0 & {$[0,0-0,0]$} & 1,7 & {$[1,7-1,7]$} & 0,8 & {$[0,8-0,8]$} \\
\hline $5-14$ años $(\mathrm{n}=15,076)$ & 0,9 & {$[0,9-0,9]$} & 1,2 & {$[1,2-1,2]$} & 1,0 & {$[1,0-1,1]$} \\
\hline 15-44 años $(n=225,747)$ & 11,0 & {$[10,9-11,1]$} & 20,7 & {$[20,6-20,8]$} & 15,5 & {$[15,5-15,6]$} \\
\hline $45-64$ años $(n=281,971)$ & 20,6 & {$[20,5-20,6]$} & 18,1 & {$[18,1-18,2]$} & 19,4 & {$[19,4-19,5]$} \\
\hline 65 años o mayores $(n=860,148)$ & 67,6 & {$[67,5-67,7]$} & 49,9 & {$[49,8-50,0]$} & 59,2 & {$[59,2-59,3]$} \\
\hline Edad desconocida $(\mathrm{n}=2,848)$ & 0,0 & & 0,4 & {$[0,4-0,4]$} & 0,2 & {$[0,2-0,2]$} \\
\hline Total $(\mathrm{n}=1,451,775)$ & 100,0 & & 100,0 & & 100,0 & \\
\hline Pearson: Uncorrected chi2 $(6)=$ & $1,20 \mathrm{e}+05$ & & & & & \\
\hline Design-based $\mathrm{F}(6,00,8,71 \mathrm{e}+06)=$ & $1,99 \mathrm{e}+04$ & $\operatorname{Pr}=$ & 0,000 & & & \\
\hline \multicolumn{7}{|l|}{ Escolaridad } \\
\hline Algún nivel educativo $(n=854,613)$ & 81,8 & {$[81,7-81,9]$} & 33,1 & {$[33,0-33,2]$} & 58,9 & {$[58,8-58,9]$} \\
\hline Sin educación $(n=254,556)$ & 18,2 & {$[18,1-18,3]$} & 16,8 & {$[16,7-16,9]$} & 17,5 & {$[17,5-17,6]$} \\
\hline Sin información $(n=342,606)$ & 0,0 & & 50,1 & {$[50,0-50,2]$} & 23,6 & {$[23,5-23,7]$} \\
\hline Total $(n=1,451,775)$ & 100,0 & & 100,0 & & 100,0 & \\
\hline Pearson: Uncorrected chi2(2) $=$ & $5,31 \mathrm{e}+05$ & & & & & \\
\hline Design-based $\mathrm{F}(2,00,2,90 \mathrm{e}+06)=$ & $2,79 \mathrm{e}+05$ & $\operatorname{Pr}=$ & 0,000 & & & \\
\hline \multicolumn{7}{|l|}{ Area de residencia } \\
\hline Cabecera Municipal $(\mathrm{n}=1,159,868)$ & 81,5 & {$[81,4-81,6]$} & 78,1 & {$[78,0-78,2]$} & 79,9 & {$[79,8-80,0]$} \\
\hline Centro Poblado $(n=80,722)$ & 5,2 & {$[5,2-5,3]$} & 5,9 & {$[5,9-6,0]$} & 5,6 & {$[5,5-5,6]$} \\
\hline Rural disperso $(\mathrm{n}=188,922)$ & 13,3 & {$[13,2-13,4]$} & 12,7 & {$[12,6-12,8]$} & 13,0 & {$[13,0-13.1]$} \\
\hline Sin información $(n=22,263)$ & 0,0 & & 3,3 & {$[3,2-3,3]$} & 1,5 & {$[1,5-1,6]$} \\
\hline Total $(n=1,451,775)$ & 100,0 & & 100,0 & & 100,0 & \\
\hline Pearson: Uncorrected chi2 $(3)=$ & $2,60 \mathrm{e}+04$ & & & & & \\
\hline Design-based $\mathrm{F}(3,00,4,36 \mathrm{e}+06)=$ & $86,950,922$ & $\operatorname{Pr}=$ & 0,000 & & & \\
\hline \multicolumn{7}{|l|}{ Regimen de seguridad social } \\
\hline Contributivo $(\mathrm{n}=527,421)$ & 43,5 & {$[43,4-43,7]$} & 28,2 & {$[28,1-28,3]$} & 36,3 & {$[36,3-36,4]$} \\
\hline Subsidiado $(n=708,197)$ & 47,7 & {$[47,5-47,8]$} & 50,1 & {$[49,9-50,2]$} & 48,8 & {$[48,7-48,9]$} \\
\hline Excepción $(n=44,439)$ & 3,9 & {$[3,9-3,9]$} & 2,1 & {$[2,1-2,2]$} & 3,1 & {$[3,0-3,1]$} \\
\hline Especial $(n=4,998)$ & 0,4 & {$[0,4-0,4]$} & 0,3 & {$[0,3-0,3]$} & 0,3 & {$[0,3-0,4]$} \\
\hline No asegurado $(n=122,725)$ & 4,5 & {$[4,5-4,6]$} & 12,9 & {$[12,8-12,9]$} & 8,5 & {$[8,4-8,5]$} \\
\hline Sin información $(n=43,995)$ & 0,0 & & 6,4 & {$[6,4-6,5]$} & 3,0 & {$[3,0-3,1]$} \\
\hline Total $(n=1,451,775)$ & 100,0 & & 100,0 & & 100,0 & \\
\hline Pearson: Uncorrected chi2 $(5)=$ & $1,07 \mathrm{e}+05$ & & & & & \\
\hline Design-based $F(5,00,7,26 \mathrm{e}+06)=$ & $2,15 \mathrm{e}+04$ & $\operatorname{Pr}=$ & 0.000 & & & \\
\hline \multicolumn{7}{|l|}{ Profesión } \\
\hline Agricultor $(\mathrm{n}=119,574)$ & 12,6 & {$[12,5-12,7]$} & 3,3 & {$[3,3-3,4]$} & 8,2 & {$[8,2-8,3]$} \\
\hline Inactivo $(n=62,955)$ & 7,0 & {$[6,9-7,0]$} & 1,4 & {$[1,4-1,4]$} & 4,3 & {$[4,3-4,4]$} \\
\hline No agricultor $(n=759,145)$ & 80,4 & {$[80,3-80,5]$} & 20,7 & {$[20,6-20,8]$} & 52,3 & {$[52,2-52,4]$} \\
\hline Sin informacion $(n=510,101)$ & 0,0 & & 74,6 & {$[74,5-74,7]$} & 35,1 & {$[35,1-35,2]$} \\
\hline Total $(n=1,451,775)$ & 100,0 & & 100,0 & & 100,0 & \\
\hline Pearson: Uncorrected chi2 $(3)=$ & $8,84 \mathrm{e}+05$ & & & & & \\
\hline Design-based $F(3,00,4,35 \mathrm{e}+06)=$ & $3,02 \mathrm{e}+05$ & $\operatorname{Pr}=$ & 0,000 & & & \\
\hline
\end{tabular}

Fuente: Elaboración propia con información del DANE 
Tabla 3. ORs ajustados por regresión logística para la determinación del riesgo ocupacional en las principales causas de muerte para el grupo agricultor en comparación con los no agricultores.

\begin{tabular}{|c|c|c|c|c|c|c|c|c|}
\hline Ocupación & & $\begin{array}{c}\text { Enfermedades } \\
\text { transmisibles }\end{array}$ & Neoplasias & $\begin{array}{c}\text { Sistema } \\
\text { circulatorio }\end{array}$ & $\begin{array}{l}\text { Afecciones } \\
\text { perinatales }\end{array}$ & $\begin{array}{c}\text { Causa } \\
\text { externa }\end{array}$ & $\begin{array}{l}\text { Demás } \\
\text { causas }\end{array}$ & $\begin{array}{c}\text { Signos, } \\
\text { síntomas y } \\
\text { afecciones } \\
\text { mal definidas }\end{array}$ \\
\hline \multicolumn{9}{|l|}{ Agricultor } \\
\hline & \multirow{2}{*}{$\mathrm{OR}^{\mathrm{a}}$} & $0,752^{* * *}$ & $1,125^{* * *}$ & $1,062^{* * *}$ & 1 & $1,227^{* * *}$ & $0,925^{* * *}$ & $1,441^{* * *}$ \\
\hline & & {$[0,731,0,774]$} & {$[1,106,1,144]$} & {$[1,047,1,077]$} & {$[1,1]$} & {$[1,195,1,261]$} & {$[0,911,0,940]$} & {$[1,373,1,512]$} \\
\hline & \multirow{2}{*}{$\mathrm{OR}^{\mathrm{b}}$} & $0,778^{* * *}$ & $1,149^{* * *}$ & $1,030^{* * *}$ & 1 & 0,981 & $0,923^{* * *}$ & $1,225^{* * *}$ \\
\hline & & {$[0,752,0,804]$} & {$[1,127,1,171]$} & {$[1,013,1,047]$} & {$[1,1]$} & {$[0,949,1,014]$} & {$[0,907,0,940]$} & {$[1,151,1,303]$} \\
\hline \multicolumn{9}{|l|}{ Inactivo } \\
\hline & \multirow{2}{*}{$\mathrm{OR}^{\mathrm{a}}$} & $0,934^{* * *}$ & $0,947^{* * *}$ & 0,987 & 0,469 & $1,456^{* * *}$ & $1,057^{* * *}$ & 0,983 \\
\hline & & {$[0,903,0,967]$} & {$[0,928,0,967]$} & {$[0,969,1,005]$} & {$[0,0648,3,398]$} & {$[1,398,1,517]$} & {$[1,038,1,078]$} & {$[0,903,1,069]$} \\
\hline & \multirow{2}{*}{$\mathrm{OR}^{\mathrm{b}}$} & $0,908^{* * *}$ & $0,961^{* * *}$ & 1,002 & 0,529 & $1,449^{* * *}$ & $1,052^{* * *}$ & 1,102 \\
\hline & & {$[0,874,0,944]$} & {$[0,941,0,983]$} & {$[0,982,1,022]$} & {$[0,0706,3,971]$} & {$[1,378,1,523]$} & {$[1,030,1,074]$} & {$[0,998,1,216]$} \\
\hline \multicolumn{9}{|c|}{ Sin información } \\
\hline & \multirow{2}{*}{$\mathrm{OR}^{\mathrm{a}}$} & $0,691^{* * *}$ & $0,715^{* * *}$ & $1,026^{* * *}$ & $0,590^{* * *}$ & $3,231^{* * *}$ & $0,821^{* * *}$ & $1,910^{* * *}$ \\
\hline & & {$[0,680,0,703]$} & {$[0,707,0,723]$} & {$[1,017,1,035]$} & {$[0,441,0,788]$} & {$[3,183,3,280]$} & {$[0,813,0,829]$} & {$[1,856,1,966]$} \\
\hline
\end{tabular}

Exponentiated coefficients; $95 \%$ confidence intervals in brackets

${ }^{*} p<0,05,{ }^{* *} p<0,01,{ }^{* * *} p<0,001$

${ }^{\text {a }}$ ORs ajustados por factores socioeconómicos y departamento habitual de residencia en la muestra total.

${ }^{\mathrm{b}}$ ORs ajustados por factores socioeconómicos y departamento habitual de residencia en la submuestra.

Acorde a las estimaciones presentadas en la Tabla 3, los agricultores, los inactivos y los individuos sin información ocupacional, tienen menor probabilidad de fallecer por una enfermedad transmisible, que por cualquier otra causa de muerte en comparación con los no agricultores. El riesgo de fallecer por una enfermedad transmisible para los agricultores es inferior $24,8 \%$ en la muestra y $22,2 \%$ en la submuestra en comparación con los no agricultores. Igualmente, para los inactivos el riesgo es inferior en $6,6 \%$ y $9,2 \%$ en la muestra y submuestra respectivamente. Con respecto al mismo grupo de referencia, los individuos sin información tienen un riesgo inferior de 30,9\%.

Para el caso de las neoplasias la ocupación de los agricultores parece ser un factor de riesgo, pues estos tienen aproximadamente $12 \%$ y $14 \%$ más de riesgo de fallecer por una neoplasia, en comparación con el grupo no agricultor en la muestra y submuestra respectivamente. Caso contrario, para los inactivos y sin información, para los cuales la ocupación es un factor protector.

Para las enfermedades del sistema circulatorio, el grupo agricultor en comparación con los no agricultores tiene mayor riesgo de fallecer por dicha causa, pues en la muestra tiene $6 \%$ más de riesgo y en la submuestra
3\%. Para los individuos sin información, dicho riesgo es superior en 2,6\%, mientras que la ocupación para el grupo inactivo no parece ser significativa.

Para el caso de las afecciones perinatales, se evidencia un error sistemático de diligenciamiento del certificado de defunción, debido a que el periodo perinatal comprende entre la semana 28 de gestación y el 7 día de nacimiento, lo que sugiere, que dicho campo ocupacional se ha llenado con la información de la madre. No obstante, la ocupación para los inactivos no es significativa y para el grupo agricultor no se presentó ningún caso. Cabe resaltar, que los individuos sin información tienen un riesgo de 59\% inferior que los no agricultores.

Para las causas externas, la ocupación de los agricultores es un factor de riesgo en la muestra total, pero un factor protector en la submuestra, dicha diferencia limita la interpretación de los resultados debido al sesgo de selección anteriormente mencionado. No obstante, el grupo inactivo presenta $45 \%$ y $44 \%$ más de riesgo en la muestra total y en la submuestra, respectivamente. Cabe destacar, que el grupo sin información es quien tiene mayor riesgo (3 veces más aproximadamente), posiblemente debido a que dicha categoría comprende ciertas causas que dificultan la identificación del individuo. 
En las demás causas de defunción, la ocupación es un factor protector para los agricultores y los individuos sin información, pues tienen aproximadamente un $8 \%$ menos de riesgo los primeros en la muestra y submuestra, y los últimos un $17,9 \%$ más de riesgo en la muestra total. Por el contrario, es un factor de riesgo para el grupo de los individuos inactivos pues en comparación con los no agricultores tienen un $5 \%$ más de riesgo ya sea en la muestra o submuestra.

Los signos, síntomas y afecciones mal definidas, son de principal interés debido a que pueden estar indicando calidad y barreras de acceso en el sistema de salud, pues esta categoría comprende contratiempos médicos, así como, complicaciones ulteriores a los que se pueden enfrentar los individuos de los diferentes grupos ocupacionales. El grupo agricultor, tiene un riesgo superior de fallecer por dicha causa de aproximadamente $44 \%$ en la muestra total y del $22 \%$ en la submuestra, en comparación con el grupo no agricultor. Igualmente, los individuos sin información tienen un riesgo superior del $91 \%$ con respecto al grupo de referencia.

\section{Discusión}

En la mayor parte de los trabajos relacionados con la mortalidad de los agricultores, se ha evaluado la posible asociación entre la profesión agrícola y la mortalidad o morbilidad por neoplasias, debido a los riesgos inherentes de la profesión; como la manipulación de ciertos plaguicidas, polvo y luz, entre otros, para el desarrollo de dichas patologías.

Un estudio retrospectivo, realizado en una región de Brasil, usando certificados de defunción para el periodo de estudio 1979-1998, encontró que la principal causa de muerte en el grupo agricultor, son las enfermedades cardiovasculares con una participación del 34,8\% seguida por las causas externas con un $18.4 \%$ y el cáncer con $10.6 \%$. Teniendo como referencia la población del área local y usando la Tasa de Mortalidad Estandarizada (TME), se observa que los agricultores entre 30 y 69 años presentan una menor mortalidad por cáncer y por enfermedades cardiovasculares, siendo solo significativa esta última. Por el contario, los agricultores presentaron mayor mortalidad por causas externas y enfermedades del sistema respiratorio siendo significativa solo la primera para el periodo de estudio ${ }^{17}$.

En 11 regiones de Francia se comparó la TME de agricultores y dueños de granjas con la población general de las mismas, obteniéndose tanto para hombres como para mujeres mayores de 18 años, menor mortalidad global en hombres (TME; 0.68 IC $95 \% 0.67-0.70$ ) y en mujeres (TME; 0.71 IC 95\% $0.69-0.73)$, de igual forma para el cáncer siendo inferior para los hombres (TME; 0.67 IC 95\% 0.650.70) que para las mujeres (TME; 0.76 IC $95 \% 0.71$ -0.80) el mismo comportamiento se observó para las enfermedades infecciosas, del sistema respiratorio y las causas externas para ambos $\operatorname{sexos}^{18}$.

Por lo general, los agricultores presentan menor mortalidad y morbilidad con respecto a la población en general ${ }^{18,19}$. No obstante, puede existir una subestimación del riesgo debido a la comparación de los agricultores con dicha población, quizá debido a mejores condiciones de salud asociadas a la mayor demanda de actividad física de las actividades agrícolas o estilos de vida más saludable, lo que se refleja en menor mortalidad en genera ${ }^{20}$. Además, es posible la existencia de un gradiente rural, que evidencia que las tasas bajas de mortalidad también se presentan en la población rural ${ }^{2,21}$.

Para Brasil, durante 1996 - 2005 y utilizando una metodología similar a la de este trabajo, se determinó el riesgo ocupacional por medio de ORs ajustados por variables socioeconómicas que los agricultores presentaban un 38\% más de riesgo (OR; 1.38 CI 95\% 1.26 - 1.51) de desarrollar cáncer de esófago que los no agricultores, igualmente cabe señalar que la educación para este estudio fue determinante, pues los agricultores iletrados presentaban un mayor riesgo significativo que los iletrados no agricultores ${ }^{22}$.

La literatura anteriormente descrita, sugiere que la determinación del riesgo ocupacional, es susceptible a condiciones socioeconómicas como la alfabetización y la ruralidad. Además, las diferencias en las actividades agrícolas de las unidades geográficas varían los tipos e intensidad de los factores de riesgo a los que se enfrentan los agricultores, para el caso colombiano acorde a cifras del DANE, el porcentaje de Producto Interno Bruto (PIB) agrícola departamental en el nacional agrícola, tiene un Coeficiente de Variación entre los departamentos para el año 2016 mayor a la unidad, lo que implica gran heterogeneidad de la magnitud de las actividades agrícolas desarrolladas en el territorio.

A pesar de que en este trabajo se estimaron ORs ajustados por variables socioeconómicas y demográficas del individuo, esto no garantiza una 
eficiencia de la estimación del riesgo, pues en general los estudios retrospectivos presentan limitaciones al utilizar solamente los certificados de defunción para determinar la causa de muerte, debido a que se corre el riesgo de contar con un sesgo de clasificación de la enfermedad, ambigüedad para la determinación de la profesión de los individuos, y el tiempo de duración en la misma ${ }^{6}$. Asimismo, se desconoce el tiempo de diagnóstico y la intensidad de la exposición a los factores de riesgo y hábitos no saludables como tabaquismo y alcoholismo ${ }^{8}$, lo que tampoco permite directamente la comparación con una cohorte más homogénea en vez de utilizar la población en general $^{23}$. Sin embargo, las limitaciones podrían suscribirse a diferencias numéricas del riesgo y no de su significancia, por lo que los resultados de este trabajo serían consistentes con la presencia del riesgo más que con su magnitud.

\section{Conclusión}

La principal causa de muerte en Colombia para el periodo de estudio (2010-216), son las enfermedades del sistema circulatorio, dichas causas también son la principal causa de muerte entre los grupos ocupacionales; agricultor, no-agricultor e inactivos. No obstante, existen diferencias en el perfil de mortalidad entre los grupos ocupacionales, lo que indica diferencias socioeconómicas entre los mismos.

Teniendo en cuenta la consistencia de los resultados tanto en la muestra como en la submuestra, los agricultores tienen un riesgo ocupacional inherente en la mortalidad por neoplasias, por enfermedades del sistema circulatorio, y en los signos y síntomas mal definidos en comparación con el grupo no agricultor. Por el contrario, tienen menor riesgo de fallecer por una enfermedad transmisible y por las demás causas con respecto al mismo grupo de referencia. Para establecer con mayor precisión el riesgo ocupacional, se requieren esfuerzos institucionales para realizar estudios prospectivos en la población de agricultores colombianos como los realizados por el Agriculture Health Study (AHS) en Estados Unidos, lo que también permitirá identificar el uso de medidas, actitudes y comportamientos, frente a los posibles factores de riesgo de los trabajadores agrícolas.

\section{Consideraciones éticas}

El presente estudio fue realizado de manera retrospectiva, sin ninguna intervención más que el análisis de datos de acceso abierto. No obstante, los autores garantizan la disponibilidad de la hoja de programación a quienes lo consideren necesario como ejercicio de rectificación de los resultados.

\section{Conflicto de interés}

Los autores no reportan ningún conflicto de interés

\section{Referencias}

1. Ragin C, Davis-Reyes B, Tadesse H, Daniels D, Bunker $\mathrm{CH}$, Jackson $\mathrm{M}$, et al. Farming, reported pesticide use, and prostate cancer. Am J Mens Health. 2013; 7(2): 102-109. doi: https://doi. org/10.1177/1557988312458792.

2. Thelin N, Holmberg S, Nettelbladt P, Thelin A. Mortality and morbidity among farmers, nonfarming rural men, and urban referents: a prospective population-based study. Int J Occup Environ Health. 2009; 15(1): 21-28. doi: https://doi. org/10.1179/107735209799449680.

3. Boccolini P de MM, Boccolini CS, Chrisman J de R, Koifman RJ, Meyer A. Non-Hodgkin lymphoma among Brazilian agricultural workers: A death certificate case-control study. Arch Environ Occup Health. 2017; 72(3): 139-144. doi: https://doi.org/1 $0.1080 / 19338244.2016 .1179167$.

4. Miranda-Filho AL, Monteiro GTR, Meyer A. Brain cancer mortality among farm workers of the State of Rio de Janeiro, Brazil: A population-based case-control study, 1996-2005. Int J Hyg Environ Health. 2012; 215(5): 496-501. doi: https://doi. org/10.1016/j.ijheh.2011.10.007.

5. Sritharan J, Demers PA, Harris SA, Cole DC, Peters $\mathrm{CE}$, Villeneuve PJ, et al. Occupation and risk of prostate cancer in Canadian men: A case-control study across eight Canadian provinces. Cancer Epidemiol. 2017; 48: 96-103. doi: https://doi. org/10.1016/j.canep.2017.04.006.

6. Depczynski J, Lower T. A review of prostate cancer incidence and mortality studies of farmers and non-farmers, 2002-2013. Cancer Epidemiol. 2014; 38(6): 654-662. doi: https://doi.org/10.1016/j. canep.2014.09.001.

7. Blair A, Zahm SH. Agricultural exposures and cancer. Environ Health Perspect. 1995; 103(Suppl. 8): 205-208. doi: https://doi.org/10.1289/ ehp.95103s 8205.

8. Zhao G, Ronda E, Cea L, Pulido J, Barrio G, Regidor E. Mortality by cause of death and risk behaviors in farmers versus non-farmers: the importance of avoiding the healthy worker effect. Int Arch Occup Environ Health. 2019; 92(4): 599-608. doi: https:// 
doi.org/10.1007/s00420-018-1396-2.

9. Alexopoulos EC, Kavalidou K, Messolora F. Suicide mortality across broad occupational groups in Greece: A descriptive study. Saf Health Work. 2016; 7(1): 1-5. doi: https://doi.org/10.1016/j. shaw.2015.09.004.

10. MacFarlane E, Benke G, Del Monaco A, Sim MR. Causes of death and incidence of cancer in a cohort of Australian pesticide-exposed workers. Ann Epidemiol. 2010; 20(4): 273-280. doi: https://doi. org/10.1016/ j.annepidem.2010.01.004.

11. Faria NMX, Fassa AG, Meucci RD. Association between pesticide exposure and suicide rates in Brazil. Neurotoxicology. 2014; 45: 355-362. doi: https://doi.org/10.1016/j.neuro.2014.05.003.

12. Arrighi HM, Hertz-Picciotto I. The evolving concept of the healthy worker survivor effect. Vol. 5, Epidemiology. Lippincott Williams \& Wilkins; p. 189-196.

13. Wen CP, Tsai SP. Anatomy of the healthy worker effect - A critique of summary statistics employed in occupational epidemiology. Scand J Trabajo Entorno Salud. 1982; 8(Suppl 1): 48-52.

14. Li CY, Sung FC. A review of the healthy worker effect in occupational epidemiology. Occup Med (Lond). 1999; 49(4): 225-229. doi: https://doi. org/10.1093/occmed/49.4.225.

15. Omran AR. The epidemiologic transition: a theory of the epidemiology of population change. Milbank Q. 2005; 83(4): 731-757. doi: https://doi. org/10.1111/j.1468-0009.2005.00398.x.

16. Salomon JA, Murray CJL. The epidemiologic transition revisited: compositional models for causes of death by age and sex. Popul Dev Rev. 2002; 28(2): 205-228.

17. Meyer A, Chrisman J, Moreira JC, Koifman S. Cancer molrtality among agricultural workers from Serrana Region, state of Rio de Janeiro, Brazil. Environment Res. 2003; 93(3): 264-271. doi: https:// doi.org/10.1016/s0013-9351 (03) 00065-3.

18. Levêque-Morlais N, Tual S, Clin B, Adjemian A, Baldi I, Lebailly P. The AGRIculture and CANcer (AGRICAN) cohort study: enrollment and causes of death for the 2005-2009 period. Int Arch Occup Environ Health. 2015; 88(1): 61-73. doi: https://doi. org/10.1007/s00420-014-0933-X.

19. Waggoner JK, Kullman GJ, Henneberger PK, Umbach DM, Blair A, Alavanja MCR, et al. Mortality in the Agricultural Health Study, 19932007. Am J Epidemiol. 2011; 173(1): 71-83. doi: https://doi.org/10.1093/aje/kwq323.

20. Blair A, Sandler DP, Tarone R, Lubin J, Thomas
K, Hoppin JA, et al. Mortality among participants in the agricultural health study. Ann Epidemiol. 2005; 15(4): 279-285. doi: https://doi.org/10.1016/j. annepidem.2004.08.008.

21. Stiernström EL, Holmberg S, Thelin A, Svärdsudd K. A prospective study of morbidity and mortality rates among farmers and rural and urban nonfarmers. J Clin Epidemiol. 2001; 54(2): 121-126. doi: https:// doi.org/10.1016/s0895-4356 (00) 00287-0.

22. Meyer A, Alexandre PCB, de Rezende Chrisman J, Markowitz SB, Koifman RJ, Koifman S. Esophageal cancer among Brazilian agricultural workers: Casecontrol study based on death certificates. Int J Hyg Environ Health. 2011; 214(2): 151-155. doi: https:// doi.org/10.1016/.ijheh.2010.11.002.

23. Liu H, Patel D, Welch AM, Wilson C, Mroz MM, $\mathrm{Li} \mathrm{L}$, et al. Association between occupational exposures and sarcoidosis. Chest. 2016; 150(2): 289-298. doi: https://doi.org/10.1016/j. chest.2016.01.020. 